Canadian University Music Review

Revue de musique des universités canadiennes

Guy Sacre. La musique de piano : dictionnaire des compositeurs et des œuvres. Collection « Bouquins ". Paris : Éditions Robert Laffont, 1998. 2 vol. Xxxviii (vol. 1, A-I), xxvi (vol. 2, J-Z), 2998 p. ISBN 2-221-05017-7, 2-221-08566-3 (couverture cartonnée).

\title{
Marc-André Roberge
}

Volume 19, numéro 1, 1998

URI : https://id.erudit.org/iderudit/1014614ar

DOI : https://doi.org/10.7202/1014614ar

Aller au sommaire du numéro

Éditeur(s)

Canadian University Music Society / Société de musique des universités canadiennes

ISSN

0710-0353 (imprimé)

2291-2436 (numérique)

Découvrir la revue

Citer ce compte rendu

Roberge, M.-A. (1998). Compte rendu de [Guy Sacre. La musique de piano : dictionnaire des compositeurs et des ouvres. Collection "Bouquins ». Paris : Éditions Robert Laffont, 1998. 2 vol. xxxviii (vol. 1, A-I), xxvi (vol. 2, J-Z), 2998 p. ISBN 2-221-05017-7, 2-221-08566-3 (couverture cartonnée).] Canadian University Music Review / Revue de musique des universités canadiennes, 19(1), 104-107. https://doi.org/10.7202/1014614ar

All Rights Reserved (C Canadian University Music Society / Société de musique des universités canadiennes, 1998
Ce document est protégé par la loi sur le droit d'auteur. L'utilisation des services d'Érudit (y compris la reproduction) est assujettie à sa politique d'utilisation que vous pouvez consulter en ligne.

https://apropos.erudit.org/fr/usagers/politique-dutilisation/ 
do not stand up to [today's] Schenkerian criticism" (p. 249). But all's well that ends well: after a highly circuitous route through numerous complex analytical graphs, Laufer reaches-with well-nigh palpable relief - the only desirable conclusion: however it may strike us, and despite anything he himself may have told his pupils, Bruckner did, in fact, compose in a manner which satisfies "Schenker's concept of the organic nature of a great work of art" (p. 255).

These proceedings conclude with Joseph C. Kraus's refreshingly brief "Phrase Rhythm in Bruckner's Early Orchestral Scherzi" (pp. 278-97), a study based on analytical principles and techniques introduced in the 1970s and 1980s by Carl Schachter and William Rothstein. Though ideas of the "normative" (such as achttaktige Periode) crop up here, too, this piece steers clear of the shoals of l'analyse pour l'analyse; accordingly, it manages to impart to the reader a genuine sense of the living music. And in today's world of leadingedge music analysis, that is not a small accomplishment.

In their Preface, the editors write: "When Anton Bruckner died in October 1896, the musical world lost a composer, theorist, teacher, and performer who stood at the cutting edge of the avant-garde of his generation" (p. xi). Such a statement would have been dismissed as hyperbole just twenty years ago. Although not free of its own kinds of excess, this book, as a whole, illuminates well the road that has brought us to recognizing such a pronouncement as worthy of serious contemplation. Aside from a few minor captioning and typographical problems, the volume is beautifully produced and richly illustrated with photographs, facsimiles, tables, and analytical graphs.

Zoltan Roman

Guy Sacre. La musique de piano : dictionnaire des compositeurs et des cuvres. Collection «Bouquins ». Paris : Éditions Robert Laffont, 1998. 2 vol. xxxviii (vol. 1, A-I), xxvi (vol. 2, J-Z), 2998 p. ISBN 2-221-05017-7, 2-221-08566-3 (couverture cartonnée).

Les musiciens connaissent les Éditions Robert Laffont pour quelques ouvrages de référence volumineux, dans plusieurs cas des traductions, comme celles du Baker's Biographical Dictionary of Musicians, du New Oxford Companion to Music et du New Kobbé's Complete Opera Book. Leur plus récent titre est un ouvrage que l'on pourra considérer, du moins à première vue, comme le pendant français du Handbuch der Klavierliteratur de Klaus Wolters (Zürich : Atlantis Verlag, 1967; $2^{\mathrm{e}}$ éd., 1977) et du Guide to the Pianist's Repertoire de Maurice Hinson (Bloomington : Indiana University Press, 1973; $2^{e}$ éd., 1987). Son auteur, Guy Sacre, que l'éditeur présente comme un compositeur et pianiste qui a exploré pendant 10 ans des milliers de partitions « afin de les rendre intelligibles à la fois pour le spécialiste et pour l'amateur » (notice publicitaire), ne semble pas avoir encore droit à une notice dans les dictionnaires spécialisés. Les quelques mentions du nom dans l'Internet nous révèlent qu'il est né en 1948 et que quelques-unes de ses pièces pour piano (Variations sur 
une mazurka de Chopin, Chansons enfantines, etc.) ont été enregistrées par un certain Billy Eidi sous étiquette Timpani 1C1026. L'apparente absence de toute affiliation universitaire et de publications savantes ne joue pas en faveur d'un auteur qui s'aventure à publier près de 3000 pages au sujet de quelque 4000 œuvres de 272 compositeurs. Le responsable de la collection, Guy Schoeller, précise que les œuvres "sont analysées et jugées, parfois sévèrement ». Il ajoute que l'ouvrage «n'est pas un simple répertoire; son auteur ne se contente pas d'être l'historien de la musique de piano; il veut faire partager au lecteur ses coups de cœur, ses enthousiasmes, son goût pour un instrument qui se prête aussi bien à la confidence qu'aux déchaînements de la passion » (notice publicitaire). En excluant l'objectivité à laquelle on s'attend d'un dictionnaire, cette attitude soulève un problème majeur propre à jeter un doute sur la qualité et la pertinence de l'ouvrage et sur lequel je reviendrai.

Chacun des deux volumes du dictionnaire, imprimé sur papier bible, commence par un répertoire alphabétique des auvres, lesquelles sont ensuite traitées de façon chronologique. L'ouvrage ne se limite pas à la musique pour piano, mais ouvre ses pages au clavecin en traitant des auvres d'une quarantaine de compositeurs nés avant 1700; la majeure partie est cependant consacrée au piano romantique, « avec ses précurseurs tâtonnants et son interminable et admirable ribambelle »(p. xii). Des 500 compositeurs dont il avait à l'origine l'intention d'explorer les œuvres, Sacre n'en a retenu que 272 pour reporter à une édition ultérieure ceux nés après 1920 . On pourra trouver qu'il accorde une trop grande importance aux compositeurs français, apparemment « par goût, par conviction » (p. $x$ ), mais il est évident qu'un auteur anglophone ou germanophone n'aurait pas consacré autant de pages à des gens comme Alain, Bréville, Cliquet-Pleyel, Grovlez, Jacob, Lesur, Migot, Roger-Ducasse et Wiéner. On se rappellera à cet égard le nombre d'obscurs organistes et chefs de chœur anglais qui peuplaient les pages de l'ancienne édition du Grove.

L'œuvre pour piano de chaque compositeur fait d'abord l'objet d'une présentation générale allant de quelques paragraphes à quelques pages. On trouve ensuite une présentation détaillée de l'ensemble des œuvres; chacune d'entre elles est habituellement précédée des renseignements suivants : date de composition, éditeur et date de publication, dédicataire et date de création. Les grands compositeurs sont évidemment traités de manière très détaillée : Bach, Beethoven, Chopin, Debussy, Liszt et Schumann reçoivent entre 51 et 104 pages (pour une moyenne de 77). Plusieurs compositeurs qui ne jouissent pas du même renom ont cependant droit à un nombre de pages assez élevé; l'auteur consacre entre 10 et 27 pages (pour une moyenne de 20) à des gens comme Arensky, Balakirev, Bax, Cui, Dussek, Glazounov, Heller, Liadov, Mompou et Schmitt. Si l'espace consacré à certains compositeurs dépasse parfois, voire souvent, leur réputation, on peut remercier Sacre de nous donner ce qui ne se trouve pas ailleurs. L'ouvrage est complété par une bibliographie de 40 pages composée d'ouvrages généraux sur le répertoire de la musique pour piano, puis d'articles et de livres sur la plupart des compositeurs représentés. On y retrouve 
principalement des titres en français et en anglais de même que plusieurs références à des thèses de doctorat américaines.

Tout ouvrage de nature encyclopédique s'expose à la critique autant pour les noms qu'il omet que pour ceux qu'il inclut. Compte tenu de la pauvreté de la littérature en français sur le sujet, il est dommage que Sacre ait fait autant de place à des noms qui ne pèsent à peu près pas dans la balance, plutôt que d'ouvrir ses pages aux compositeurs nés après 1920, comme Xenakis, Ligeti, Berio, Boulez, Stockhausen, Stevenson, Crumb, Bolcom et Rzewski. Le choix aurait été ardu, sans doute, mais il est difficilement justifiable de passer ainsi à côté de gens qui ont apporté à la littérature de l'instrument une contribution notable. Plusieurs noms manquent parmi les compositeurs nés avant 1920; si Sacre retient Alkan, Liapounov, Busoni, Reger et Medtner, il ignore Tausig, Moszkowski, Godowsky, Grainger, Sorabji, Korngold, Antheil, Krenek, Wolpe, Cage et Nancarrow.

Dans son introduction, Sacre annonce que, pour « réparer ce que certains auront pris hâtivement pour de la désinvolture ", il travaille à un volume complémentaire comprenant des aperçus et des tableaux illustrant « une histoire de l'instrument et du répertoire, un abrégé de la technique pianistique, un lexique des formes, une étude des thèmes, voire un panorama des virtuoses » (p. xv). La facture des volumes parus donne à penser que l'auteur ferait mieux de s'arrêter ici, car leur lecture est difficilement supportable. L'ouvrage n'a rien à voir avec la musicologie moderne, mais appartient au type de musicographie « enrubannée » qui se pratiquait en France pendant la première moitié du siècle, comme cette description d'un motet de Dunstable que faisait André Pirro (1869-1943) dans son Histoire de la musique de la fin du XIVe siècle à la fin du XVIe (Paris : Henri Laurens, 1940), p. 98 : «Il ne veut se mouvoir que par sauts et par bonds, quand les compositeurs s'abandonnent encore si volontiers au courant de la gamme. À leurs descentes résignées, il préfère les chutes hasardeuses, et il se lance de même à l'escalade, avec un mépris insolent de la gradation. "

Pour Sacre, la sonate est un "vaste sac où ne s'engouffre souvent que du vent " et son admiration complète va à la petite pièce pour piano, "le beau rassemblé dans un espace exigu, dans un cercle étroit de certitudes », qui lui « cause au cœur autant qu'à l'esprit une émotion violente » (p. xiv). Cette attitude l'amène à rédiger des descriptions qui, même si elles font souvent appel à un vocabulaire musical, ne procèdent que par allusions littéraires et par images. L'ouvrage prend malheureusement la forme d'un verbiage incontrôlable, d'une logorrhée, où l'on sent constamment la mièvrerie et le sentimentalisme. On pourrait remplir des pages en mettant bout à bout les expressions utilisées : broderies fantasques, oppressantes syncopes, gruppettos rageurs arrachés au clavier, tierces caressantes, tournoyantes volutes, croches froufroutantes, motif mélodique nimbé de tendresse, ruban de croches murmurantes, pimpante petite fanfare, cascatelles de doubles notes, triolets de croches songeuses, trilles qui vibrent comme des élytres d'insectes. Le Nocturne en fa dièse mineur, op. $48, n^{\circ} 2$, de Chopin possède un « contrepoint si ductile, fruit 
d'une oreille infaillible, [qui] est mué aussitôt en pure poésie. La beauté de certaines modulations [...] fait presque monter les larmes aux yeux » (p. 690). Au sujet d' "Ondine », le huitième des Préludes du deuxième livre de Debussy, on lit :

Ondine, fée industrieuse, règle à l'équerre et au métronome le ballet des fontaines, des cascades, des geysers. Mais hormis quelques éclaboussures d'arpèges, quelques traits scintillants, le prélude debussyste n'est pas particulièrement aquatique; et c'est une humoresque de plus; cette Ondine-ci, à la manière de Puck et des « exquises danseuses », assemble capricieusement les motifs, les combine, les défait, dans un ravissant et poétique désordre ( $p$. 929-30).

Compte tenu de l'attitude qui a présidé à la recherche et à la rédaction, on peut facilement s'imaginer que Sacre n'hésite pas à recourir aux jugements de valeur. La Sonate en la mineur, op. 38, $\mathrm{n}^{\circ} 1$ (« Reminiscenza »), de Medtner lui inspire le commentaire suivant:

Mais elles [les quatre lignes de prologue] donnent leur ton à toute la sonate, et qu'on les juge ou non touchantes (en réalité, elles ne sont que mièvres), elles déteignent par trop sur le restant : lieux communs, lyrisme amorphe, on se lasserait vite (et la pièce a beau ne durer qu'un quart d'heure, on trouverait le temps long), n'était un passage singulier du développement, au trouble ondoiement chromatique, qui ressemble à du Prokofiev (p. 1818).

Le type de musicographie que pratique Guy Sacre risque fort d'être perçu avec beaucoup de méfiance par les gens avertis, qui seraient forcés de perdre un temps précieux pour isoler les faits du fatras pseudo-poétique qui enrubanne les descriptions. Certains amateurs trouveront peut-être quelque plaisir à savoir ce que Guy Sacre pense de la littérature du piano, mais les autres risquent fort de regretter une dépense de 100 dollars.

Sacre a de toute évidence fourni une somme de travail gigantesque, ne serait-ce que pour rassembler et consulter autant de partitions et les lire au piano (car nombre d'entre elles n'existent pas sur disque), et ce, sans parler de la rédaction. Il aurait dû faire un tri beaucoup plus serré et aller droit au but, sans chercher à jouer au poète. Devant ces 3000 pages de « coups de cœur » et d'épanchements émotifs, on ne peut résister à la tentation de retourner contre Sacre cette phrase de Debussy placée en épigraphe : « [J]e veux avoir la liberté de dire qu'une page ennuyeuse m'ennuie, quel que soit son auteur. »

Marc-André Roberge 